\title{
Identification of a Nicotinic Acetylcholine Receptor on Neurons Using an $\alpha$-Neurotoxin That Blocks Receptor Function
}

\author{
Stanley W. Halvorsen and Darwin K. Berg \\ Department of Biology, University of California, San Diego, La Jolla, California 92093
}

\begin{abstract}
An $\alpha$-neurotoxin, Bgt 3.1, that reversibly blocks the ACh response of chick ciliary ganglion neurons has heen used to identify 2 classes of high-affinity binding sites on the cells in culture. The first class appears to be the $\alpha$-bungarotoxin binding site on the neurons. The second class of Bgt 3.1 sites is distinct from the $\alpha$-bungarotoxin binding sites and has the properties expected for the functional nicotinic $\mathrm{ACh}$ receptor on the cells. Equilibrium binding and kinetic studies indicate a $K_{d}$ value of 5-6 nM for Bgt 3.1 at the second class of sites. The kinetics and affinity of binding are consistent with those inferred from previous physiological studies for Bgt 3.1 inhibition of receptor function. Bgt 3.1 binding to the sites is completely inhibited by each of the cholinergic ligands $\mathrm{ACh}$, carbachol, nicotine, $d$-tubocurarine, and trimethaphan, but not by $\alpha$-bungarotoxin. Highest site densities are found in cultures of ciliary and sympathetic ganglion neurons, cell types known to have ganglionic nicotinic ACh receptors. Low levels of sites may be present in cultures of spinal cord and dorsal root ganglion neurons; no binding is found in cultures of skeletal myotubes or cardiac cells when $\alpha$-bungarotoxin is used to block Bgt 3.1 binding to $\alpha$-bungarotoxin sites. These results demonstrate that Bgt 3.1 can be used as a specific probe for the nicotinic $\mathrm{ACh}$ receptor on chick autonomic neurons.
\end{abstract}

Neuronal nicotinic acetylcholine receptors (AChRs) mcdiatc the primary chemical synaptic transmission through ganglia of the autonomic nervous system and play important roles in the CNS as well. Though neuronal AChRs are similar in many physiological respects to the well-characterized nicotinic AChRs of vertebrate muscle and electric organ, physical characterization of neuronal AChRs has been difficult to interpret because of controversy over suitable molecular probes. $\alpha$-Bungarotoxin $(\alpha-$ $\mathrm{Bgt}$ ), a protein neurotoxin that has proved valuable in studying muscle and electric organ AChRs, has also been suggested as a probe for neuronal AChRs. Recently, $\alpha$-Bgt binding components have been purified from locust nervous system (Breer et al., 1985) and from chick (Conti-Tronconi et al., 1985) and rat brain (Kemp et al., 1985), and characterized as possible nicotinic AChRs. Partial amino acid sequence data from the chick brain component indicate sequence homology with the AChR from chick muscle. The failure of $\alpha$-Bgt in most instances to block vertebrate neuronal $\mathrm{ACh}$ responses, however, in contrast to its efficient blockade of muscle AChR responses, has left questions as to the function of the $\alpha$-Bgt binding component on neurons.

\footnotetext{
Received Feb. 18, 1986; revised May 1, 1986; accepted May 6, 1986.

We thank Dannielle Pellegrin for expert technical assistance, and Alfredo Franco, Jr., for help with preparing the figures. Grant support was provided by the National Institutes of Health (NS 12601), the Muscular Dystrophy Association, and the American Heart Association, with funds contributed in part by the California Heart Association. S.W.H. is a Muscular Dystrophy Postdoctoral Fellow.

Correspondence should be addressed to Darwin K. Berg, Department of Biology, B-022, University of California, San Diego, La Jolla, CA 92093.

Copyright (C) 1986 Society for Neuroscience $0270-6474 / 86 / 113405-08 \$ 02.00 / 0$
}

Studies on the rat pheochromocytoma cell line PC1 2 (Mitsuka and Hatanaka, 1983; Patrick and Stallcup, 1977) and on chick ciliary ganglion neurons (Jacob and Berg, 1983; Jacob et al., 1984; Smith et al., 1983) indicate that the membrane component responsible for the fast, depolarizing response attributed to nicotinic AChRs on the cells is clearly distinct from the component on the same cells that binds $\alpha$-Bgt. Moreover, recent studies on detergent extracts prepared from chick and rat brain show that most, if not all, of the high-affinity binding for cholinergic agonists occurs with components that can be physically separated from those binding $\alpha$-Bgt (Schneider et al., 1985; Whiting and Lindstrom, $1986 \mathrm{a}, \mathrm{b})$. These results underscore the value of developing new probes that identify functional nicotinic AChRs in the vertebrate nervous system and suggest caution in attributing, at this time, a specific functional role to the $\alpha$-Bgt binding component found on neurons.

Monoclonal antibodies (mAbs) to the "main immunogenic region" (MIR) of muscle and electric organ AChR $\alpha$-subunit have been used to identify a membrane component on chick ciliary ganglion neurons that is a strong candidate for the functional, synaptic AChR on the cells. The component is concentrated in synaptic membrane (Jacob et al., 1984), has biochemical properties expected for an AChR (Smith et al., 1985), can be modulated by exposure of the cells to cholinergic ligands (Smith et al., 1986), and is clearly distinct from the $\alpha$-Bgt binding component on the cells, which appears to be confined to extrasynaptic regions (Jacob and Berg, 1983; Jacob et al., 1984; Smith et al., 1983, 1985). The same anti-MIR mAbs have been used to purify a component from chick brain that binds nicotine but not $\alpha$-Bgt (Whiting and Lindstrom, 1986a, b). Antisera to the brain component specifically block the nicotinic $\mathrm{ACh}$ response of chick ciliary ganglion neurons in cell culture (Stollberg et al., 1986). Taken together, these findings strongly suggest that the brain and ganglionic components recognized by the anti-MIR $\mathrm{mAbs}$ are functional neuronal nicotinic AChRs. Two concerns remain. First, the cross-reacting $m A b s$ currently available do not alter receptor function either for neuronal $\mathrm{AChR}$ or muscle $A C h R$, preventing a definitive and direct confirmation of receptor identity on neurons using the mAbs alone. Second, though in most instances comodulation is seen for the number of putative AChRs recognized by the mAbs and the relative levels of $\mathrm{ACh}$ response associated with the neurons as predicted, in at least one instance a significant unexplained discrepancy remains: One growth condition in culture produces a 3- to 4-fold change in the levels of $\mathrm{ACh}$ sensitivity that develop without producing a corresponding change in the levels of surface component detected by anti-MIR mAbs (Margiotta et al., 1985, and unpublished observations; Smith et al., 1986).

An alternative approach to identifying neuronal AChRs is to utilize agents known to block receptor function. Bgt 3.1 is an $\alpha$-neurotoxin present as a minor component in the venom of Bungarus multicinctus. The toxin can be separated from $\alpha$-Bgt, a major component in the same venom, and, unlike $\alpha$-Bgt, Bgt 
3.1 specifically and reversibly blocks the $\mathrm{ACh}$ response of chick ciliary ganglion neurons in cell culture (Ravdin and Berg, 1979). Indirect studies have suggested that ciliary ganglion neurons have at least 2 classes of Bgt 3.1 binding sites, one being the $\alpha$-Bgt sites on the cells and the other being the functional nicotinic AChRs (Ravdin et al., 1981). Electrophoretic and amino acid sequence analyses (Loring et al., 1986) indicate that Bgt 3.1 is indistinguishable from $\kappa$-bungarotoxin (Chiappinelli, 1983) and toxin $\mathrm{F}$ (Loring et al., 1984), all derived from the same venom. The toxin has a molecular weight of 7300 and is composed of 66 amino acids (Loring et al., 1986). Binding studies with iodinated derivatives of toxin $F$ and $\kappa$-bungarotoxin have found that only a portion of the specific binding in chick ciliary ganglia can be blocked by $\alpha$-Bgt, again suggesting 2 classes of sites (Chiappinelli, 1983; Loring et al., 1984). We have examined the binding of ${ }^{125} \mathrm{I}-\mathrm{Bgt} 3.1$ to chick ciliary ganglion neurons in dissociated cell culture and have identified a class of binding sites that exhibits the kinetic and pharmacological properties predicted for the nicotinic AChR on the neurons.

\section{Materials and Methods}

\section{Cell cultures}

Dissociated ciliary ganglion neuron cultures were prepared from $8 \mathrm{~d}$ chick embryos and grown at $37^{\circ} \mathrm{C}$ in an atmosphere of $5 \% \mathrm{CO}_{2} / 95 \%$ air on a substratum of collagen and lysed fibroblasts as previously described (Nishi and Berg, 1981). Cultures contained about $1.4 \times 10^{4}$ neurons in $16 \mathrm{~mm}$ wells and were grown in a culture medium consisting of Eagle's minimal essential medium containing $10 \%$ (vol/vol) heatinactivated horse serum, $50 \mathrm{units} / \mathrm{ml}$ penicillin, $50 \mu \mathrm{g} / \mathrm{ml}$ streptomycin, and 3\% (vol/vol) embryonic eye extract (Nishi and Berg, 1981). Culture medium was replaced at 2-3 d intervals, and cultures were taken for experiments at $7 \mathrm{~d}$.

Dissociated dorsal root ganglion (DRG) cell cultures were prepared from $9-10 \mathrm{~d}$ chick embryos by the same procedure as described for ciliary ganglion cultures except that cells were plated at an initial neuron density of $2-3 \times 104 / 16 \mathrm{~mm}$ well and the medium was supplemented with $0.4 \mu \mathrm{g} / \mathrm{ml}$ 7S NGF. Dissociated sympathetic neuron cultures were prepared from $9 \mathrm{~d}$ embryonic chick sympathetic chain ganglia and maintained as described for DRG cell cultures except that initial plating densities were $0.5-1 \times 10^{5}$ neurons $/ 16 \mathrm{~mm}$ well. Cultures of dissociated spinal cord cells were prepared from $7 \mathrm{~d}$ embryonic chick, maintained in medium supplemented with embryo extract instead of embryonic eye extract, and treated with cytosine arabinoside as previously described (Berg, 1978), using initial cell densities of $1-2.5 \times 10^{5}$ neurons/ $16 \mathrm{~mm}$ well.

Skeletal muscle myotube cultures were prepared from $11 \mathrm{~d}$ embryonic pectoral muscle as previously described (Nishi and Berg, 1977) on the same substratum as ciliary ganglion cultures. Cells were plated at an initial density of $10^{5}-10^{6}$ cells $/ 16 \mathrm{~mm}$ well, exposed to $10^{-5} \mathrm{M}$ cytosine arabinoside for a $36-48 \mathrm{hr}$ period on the second and third days of incubation, and taken for experiments on day 7.

Cardiac muscle cultures were prepared as previously described (Nathanson, 1983) from $8 \mathrm{~d}$ embryonic chick heart and plated at a density of $1-2 \times 10^{5}$ cells $/ 16 \mathrm{~mm}$ well on the substratum described for ciliary ganglion cultures. Cardiac cultures received medium of the same composition as that described for skeletal muscle cultures and were taken for experiments when the cells reached confluency at $3 \mathrm{~d}$.

\section{Protein neurotoxins}

The $\alpha$-neurotoxins Bgt 3.1 and $\alpha$-Bgt were purified from Bungarus $m u l$ ticinctus venom as previously described (Ravdin et al., 1981). Iodination of Bgt 3.1 was carried out using a modified Chloramine T method as previously described (Lindstrom et al., 1981), yielding initial specific activities of $0.5-1.1 \times 10^{18} \mathrm{cpm} / \mathrm{mol}$ and, on average, $0.2 \mathrm{~mol}$ iodine/ mol toxin.

\section{Binding assays}

Toxin binding to intact cells in culture was carried out by first replacing the culture medium with $0.2 \mathrm{ml} 37^{\circ} \mathrm{C}$ medium of the composition used for ciliary ganglion neuron cultures but containing $10^{-6} \mathrm{M} \alpha$-Bgt unless otherwise indicated. After returning the cells to the culture incubator for $15-20 \mathrm{~min}$, the binding reaction was initiated by adding $0.025 \mathrm{ml}$ of ${ }^{125}$ I-Bgt 3.1 from a stock solution. The incubation was continued for an additional 45-60 min (unless otherwise stated) and then terminated by aspirating the toxin-containing medium and rinsing the cultures rapidly 4 times with $1 \mathrm{ml}$ aliquots of warm rinse buffer consisting of $137 \mathrm{~mm} \mathrm{NaCl}, 5.4 \mathrm{~mm} \mathrm{KCl}, 0.8 \mathrm{mM} \mathrm{MgSO}_{4}, 0.9 \mathrm{mM} \mathrm{Na}_{2} \mathrm{PO}_{4}, 0.4 \mathrm{~mm}$ $\mathrm{K}_{2} \mathrm{PO}_{4}, 1.8 \mathrm{mM} \mathrm{CaCl}_{2}, 2 \mathrm{mg} / \mathrm{ml} \mathrm{BSA}, 5.6 \mathrm{~mm}$ glucose, and $5 \mathrm{~mm}$ HEPES, $\mathrm{pH}$ 7.4. The total rinse time was confined to a $10 \mathrm{sec}$ period unless otherwise indicated. The cultures were then scraped in $0.5 \mathrm{ml}$ of $0.6 \mathrm{~N}$ $\mathrm{NaOH}$ and analyzed for bound radioactivity with a Tracor analytic model 1191 gamma counter at a counting efficiency of 75\%. Data represent the mean $\pm \mathrm{SE}$ for triplicate cultures unless otherwise indicated. Specific ${ }^{125} \mathrm{I}$-Bgt 3.1 binding was determined as the amount of binding inhibited by an excess of unlabeled Bgt 3.1 (usually $1 \mu \mathrm{M}$ ).

Rapid release of ${ }^{125} \mathrm{I}$-Bgt 3.1 bound to the surface of ciliary ganglion neurons was achieved by using a mild acid rinse procedure previously described that releases a variety of protein ligands from their receptors (Ascoli, 1982; Mahan et al., 1985). Ciliary ganglion cultures were labeled with ${ }^{125} \mathrm{I}-\mathrm{Bgt} 3.1$ as indicated, rinsed and after $2 \mathrm{hr}$ at $37^{\circ} \mathrm{C}$, cooled on ice, and then incubated for $15 \mathrm{~min}$ on ice in $0.1 \mathrm{M}$ glycine, $\mathrm{pH} 2.5$, containing $50 \mathrm{~mm} \mathrm{NaCl}$ and $2 \mathrm{mg} / \mathrm{ml} \mathrm{BSA}$. The cultures were then washed with rinse buffer and assayed for bound radioactivity.

\section{Lactic acid dehydrogenase assay}

Lactic acid dehydrogenase (LDH), a relatively ubiquitous cytoplasmic enzyme, was assayed as a means of comparing relative amounts of cytoplasmic volume among cultures as previously described (Nishi and Berg, 1981). LDH activity was determined by taking aliquots from triplicate cultures grown in parallel with those used for binding studics. Cultures were solubilized with $0.1 \mathrm{ml}$ of Triton X-100 buffer consisting of $200 \mathrm{~mm} \mathrm{NaCl}, 50 \mathrm{~mm} \mathrm{NaPO}, 5 \mathrm{mg} / \mathrm{ml} \mathrm{BSA}$, and $0.5 \%$ Triton X$100, \mathrm{pH} 7.5$, and were centrifuged to remove particulate debris. $\mathrm{LDH}$ activity was measured spectrophotometrically by following the conversion of NADH to NAD in the presence of pyruvate and cell culture extract at room temperature. One unit of $\mathrm{LDH}$ activity is defined as a decrease of 1 OD unit/min in the absorption of light at $340 \mathrm{~nm}$ using a pathlength of $1 \mathrm{~cm}$.

\section{Materials}

White Leghorn chick embryos were obtained locally and maintained at $39^{\circ} \mathrm{C}$ in a humidified incubator. Tissue extracts and culture media were prepared as previously described (Nishi and Berg, 1981). $\mathrm{Na}^{125} \mathrm{I}$ was obtained from Amersham, Bungarus multicinctus venom from Miami Serpentarium, culture media components from Grand Island Biological Co., and 7S NGF from Sigma (8384). Other chemicals were obtained as previously described (Smith et al., 1986).

\section{Results}

\section{Classes of Bgt 3.1 binding sites}

Previous physiological evidence suggested that Bgt 3.1 interacts with at least 2 distinct classes of sites on chick ciliary ganglion neurons, one being a class of sites that inhibits $\alpha$-Bgt binding and the other being a class of sites that blocks ACh receptor function (Ravdin et al., 1981). To test this directly, cultures of ciliary ganglion neurons were incubated with ${ }^{125} \mathrm{I}-\mathrm{Bgt} 3.1$ in the presence of a range of $\alpha$-Bgt concentrations and examined for binding of radioactivity. $\alpha$-Bgt at $10^{-7} \mathrm{M}$ inhibited half of the total binding observed with ${ }^{125} \mathrm{I}-\mathrm{Bgt} 3.1$, and no further inhibition was obtained with $\alpha$-Bgt concentrations as high as $3 \times$ $10^{-6} \mathrm{M}$ (Fig. 1). Addition of unlabeled Bgt 3.1 at $10^{-6} \mathrm{M}$ in the presence of $10^{-6} \mathrm{M} \alpha$-Bgt inhibited an additional $25 \%$ of the total binding, or $50 \%$ of the remaining binding (Fig. 1). Thus, three fourths of the total binding under these conditions represented specific, saturable ${ }^{125}$ I-Bgt 3.1 binding in the cultures, and of this, two thirds was associated with the $\alpha$-Bgt site since it could be blocked by $\alpha$-Bgt. One third of the specific ${ }^{125}[-B g t$ 3.1 binding involved a separate class of sites since it was not blocked by $\alpha$-Bgt. In all subsequent experiments, $10^{-6} \mathrm{M} \alpha$-Bgt was routinely included in the binding reaction to block ${ }^{125}$ I-Bgt 3.1 binding to the high-affinity $\alpha$-Bgt sites. This strategy facil- 


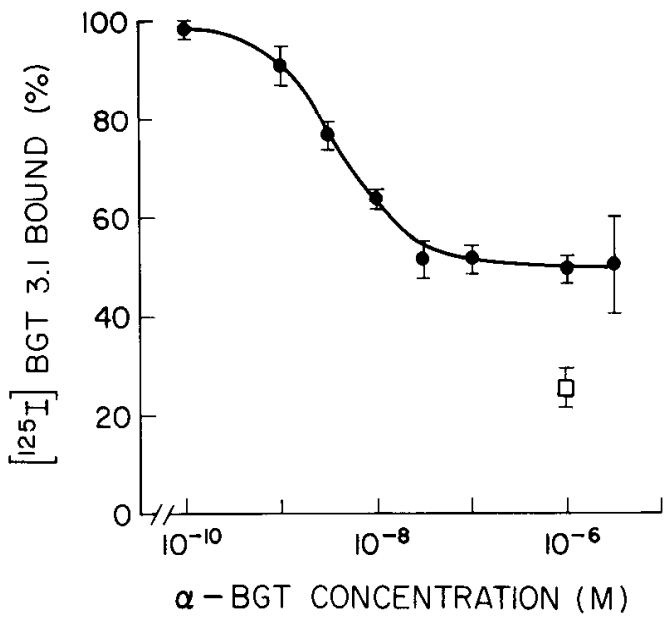

Figure 1. Partial blockade of Bgt 3.1 binding by $\alpha$-Bgt. Binding of ${ }^{125} \mathrm{I}-$ Bgt 3.1 was determined for cultures of ciliary ganglion neurons in the presence of the indicated concentrations of $\alpha$-Bgt (filled circles) and is expressed as a percentage of total binding in the absence of $\alpha$-Bgt. Unlabeled Bgt 3.1 at $10^{-6} \mathrm{M}$ was included with the $\alpha$-Bgt in one set of cultures (open box). The unlabeled toxin(s) was present both during an initial 15 min preincubation period and during the subsequent $1 \mathrm{hr}$ labeling with $10^{-8} \mathrm{M}^{125} \mathrm{I}$-Bgt 3.1. Total binding (100\%) was $6.2 \pm 0.2$ $\mathrm{fmol} /$ culturc. Similar results werc obtained in 2 other experiments.

itated characterization of the Bgt 3.1-unique sites described below

\section{Bgt 3.1-unique sites}

Specific binding of ${ }^{125} \mathrm{I}$-Bgt 3.1 in the presence of $\alpha$-Bgt reached equilibrium within $45 \mathrm{~min}$ (Fig. $2 A$ ). Kinetic analysis of the data, assuming a reversible second-order reaction (Fields et al., 1978), indicated that the rate of binding was well described by a single rate constant of $9.3 \times 10^{6} \mathrm{M}^{-1} \mathrm{~min}^{-1}$. The amount of binding was proportional to the number of neurons present over the range of $0-2$ dissociated ganglia per culture; at higher densities, the binding did not always increase proportionately (Fig. $2 B$ ). Near-saturation of the Bgt 3.1-unique sites, i.e., toxin sites not recognized by $\alpha$-Bgt, occurred under standard conditions ( 1 hr at $37^{\circ} \mathrm{C}$ ) with $10 \mathrm{~nm}{ }^{125} \mathrm{I}$-Bgt 3.1 (Fig. 3). Scatchard analyses of the data from 3 such experiments indicated a mean equilibrium dissociation constant $\left(K_{\mathrm{d}}\right)$ of $5.3 \pm 0.3 \times 10^{-9} \mathrm{M}$ and an average maximum binding $\left(B_{\text {max }}\right)$ of $2.9 \pm 0.3 \mathrm{fmol} /$ culture for cultures prepared with 2 dissociated ganglia per culture well. An example is shown in the inset of Figure 3. Only a single class of high-affinity Bgt 3.1-unique sites was observed.

The $K_{\mathrm{d}}$ for ${ }^{125} \mathrm{I}-\mathrm{Bgt} 3.1$ binding determined here agrees well with the concentrations of Bgt 3.1 needed for physiological blockade of the $\mathrm{ACh}$ response: $10^{-8} \mathrm{M} \mathrm{Bgt} 3.1$ blocks $90 \%$ of the response, while $10^{-7} \mathrm{M}$ blocks $99 \%$ (Ravdin and Berg, 1979). Similarly, the time course of receptor blockade by Bgt 3.1, i.e., within $60 \mathrm{~min}$ at $37^{\circ} \mathrm{C}$ (Ravdin and Berg, 1979), is consistent with the rate of ${ }^{125} \mathrm{I}-\mathrm{Bgt} 3.1$ binding shown here (Fig. $2 A$ ). To extend this correlation, we also examined the rate of ${ }^{125}$ I-Bgt 3.1 dissociation. Previous physiological studies indicated that at least two thirds of Bgt 3.1-induced receptor blockade can be reversed by $1-2 \mathrm{hr}$ of rinsing at $37^{\circ} \mathrm{C}$ (Ravdin and Berg, 1979). When cultures were labeled with ${ }^{125} \mathrm{I}$-Bgt 3.1 in the presence of $10^{-6} \mathrm{M} \alpha$-Bgt for $1 \mathrm{hr}$ and rinsed, the majority of binding to Bgt 3.1-unique sites was reversed within a 2 hr period (Fig. 4), as predicted by the physiological studies. The dissociation was complex, however, and was better described by biphasic rather than monophasic kinetics. Kinetic analysis indicated that $46 \%$ of the ${ }^{125} \mathrm{I}$-Bgt 3.1 binding dissociated with a rate constant of $5.2 \times 10^{-2} \mathrm{~min}^{-1}$ (fast), while the remainder dissociated with a

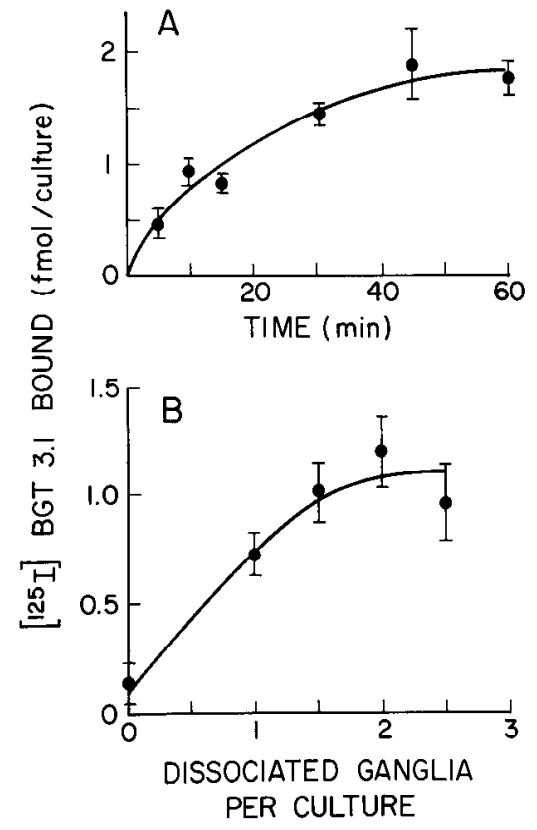

Figure 2. Time dependence and proportionality to number of neurons for Bgt 3.1 binding. Specific binding of ${ }^{125} \mathrm{I}$-Bgt 3.1 was determined in cultures of ciliary ganglion neurons under standard conditions, i.e., $10^{-8}$ $\mathrm{M}^{125} \mathrm{I}$-Bgt 3.1 for $1 \mathrm{hr}$ at $37^{\circ} \mathrm{C}$ in the presence of $10^{-6} \mathrm{M} \alpha$-Bgt with cultures containing 2 dissociated ganglia (ca. $7 \times 10^{3}$ neurons/ganglion) per culture, except for the indicated variations. $A$, Time of ${ }^{125}$ I-Bgt 3.1 binding was varied as indicated. $B$, Number of dissociated ganglia used to prepare the cultures was varied as indicated. Similar results were obtained in 2 other experiments.

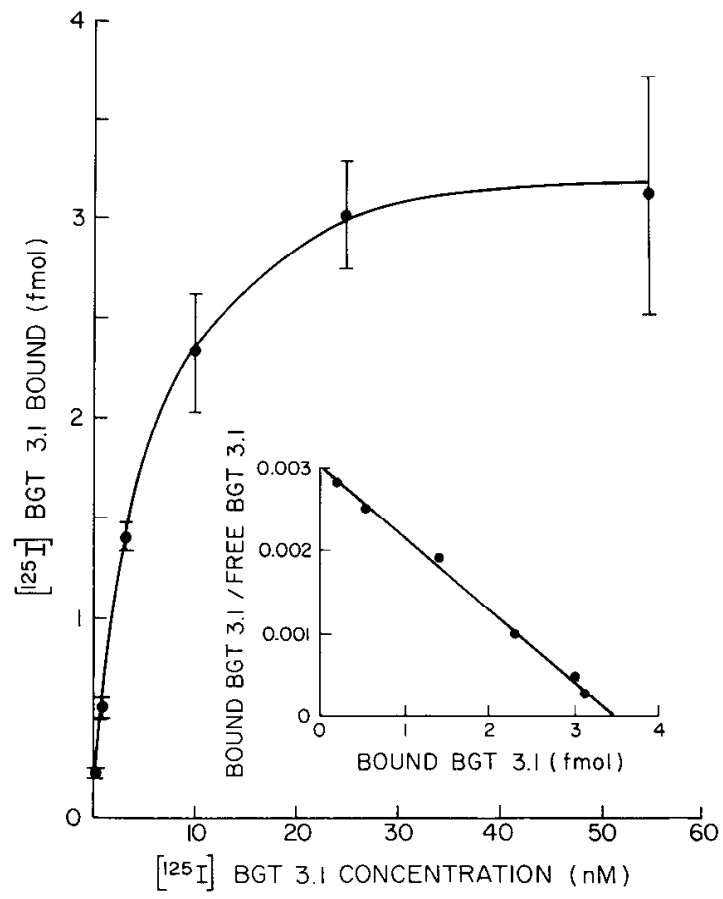

Figure 3. Concentration dependence of Bgt 3.1 binding. Cultures of ciliary ganglion neurons were incubated for $1 \mathrm{hr}$ with the indicated concentrations of ${ }^{125} \mathrm{I}$-Bgt 3.1 in the presence of $10^{-6} \mathrm{M} \alpha$-Bgt and analyzed for specific binding of Bgt 3.1. Scatchard analysis of the data (inset) indicated a $K_{\mathrm{d}}$ of $5.2 \times 10^{-9} \mathrm{M}$ and a $B_{\max }$ of $3.5 \mathrm{fmol} /$ culture for this experiment. Similar results were obtained in 2 other experiments. 


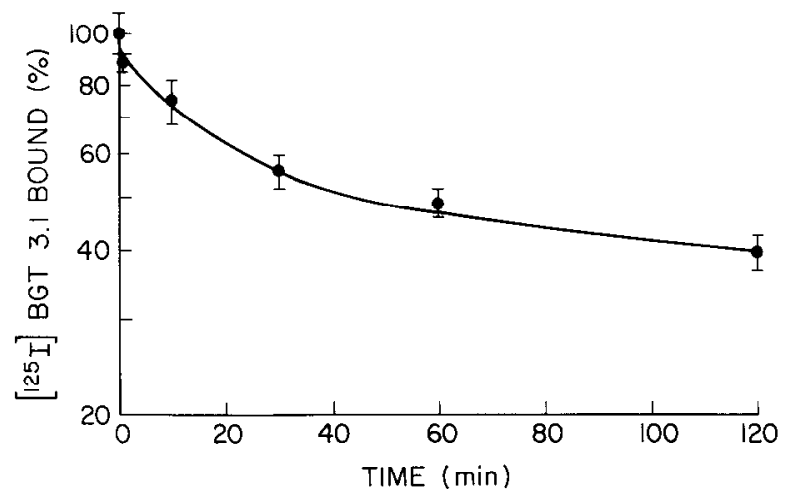

Figure 4. Rate of Bgt 3.1 receptor dissociation. Ciliary ganglion cultures were labeled with $10^{-8} \mathrm{M}{ }^{125} \mathrm{I}-\mathrm{Bgt} 3.1$ for $1 \mathrm{hr}$ at $37^{\circ} \mathrm{C}$ in the presence of $10^{-6} \mathrm{M} \alpha$-Bgt, rinsed rapidly 3 times, incubated in medium at $37^{\circ} \mathrm{C}$ for the indicated time, rinsed a final time, and analyzed for specific ${ }^{125} \mathrm{I}-$ Bgt 3.1 binding. Results are expressed on a log scale as a percentage of the specific binding observed after 4 rapid washes at zero time; the $100 \%$ value represents $1.7 \mathrm{fmol} /$ culture. The curve was drawn from a leastsquares regression fit of the data assuming 2 independent rates of dissociation. $F$ test analysis indicated that the data were fit better to a biphasic model than to a monophasic model $(p<0.05)$. Similar results were obtained in a second experiment.

rate constant of $2.3 \times 10^{-3} \mathrm{~min}^{-1}$ (slow). The fast dissociation rate constant, together with the association rate constant, yield a value for the $K_{\mathrm{d}}$ of $5.6 \times 10^{-9} \mathrm{M}$, which is in close agreement with the value calculated above from equilibrium binding data.

In principle, a multiphasic dissocation curve could arise from any of several causes, including heterogeneity of binding sites, Bgt 3.1-mediated induction of a slowly dissociating toxin-receptor complex in the plasma membrane, and internalization of bound ${ }^{125}$ I-Bgt 3.1 by the cells. To detect possible heterogeneity of binding sites, the proportion of bound ${ }^{125}$ I-Bgt 3.1 that slowly dissociates was compared for cultures labeled to different extents. Varying the concentration of ${ }^{125} \mathrm{I}$-Bgt 3.1 from 1 to 10 nM in the initial 45 min incubation resulted in a nearly 4 -fold difference in the levels of specific binding achieved at equilibrium but produced no differences in the subsequent dissociation profiles (data not shown).

$\alpha$-Bgt bound to AChR of muscle and electric organ has been shown to induce a slowly dissociating form of the toxin-receptor complex (Hess et al., 1975). To determine whether Bgt 3.1 induces conversion of the toxin-receptor complex on ciliary ganglion neurons from a fast-dissociating form to a slowly dissociating one, cultures were labeled with ${ }^{125}$ I-Bgt 3.1 to equivalent extents but for different lengths of time and were then examined for the proportion of bound ${ }^{125} \mathrm{I}-\mathrm{Bgt} 3.1$ that dissociated slowly from the cells. ${ }^{125} \mathrm{I}-\mathrm{Bgt} 3.1$ at $30 \mathrm{nM}$ was used for the labeling reaction so that complete binding was achieved during the short

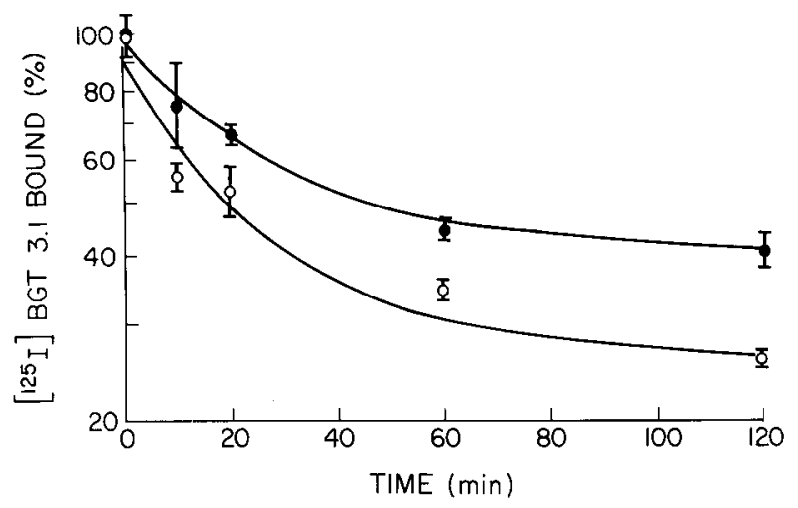

Figure 5. Effect of incubation time on dissociation kinetics. Ciliary ganglion neurons were labeled with $3 \times 10^{-8} \mathrm{M}^{125} \mathrm{I}-\mathrm{Bgt} 3.1$ in the presence of $10^{-6} \mathrm{M} \alpha$-Bgt either for $10 \mathrm{~min}$ (open circles) or for $2 \mathrm{hr}$ (closed circles), and then rinsed and examined for dissociation of specifically bound ${ }^{125} \mathrm{I}$-Bgt 3.1 as described in Figure 4 . The $100 \%$ control value obtained following a $1 \mathrm{~min}$ rinse at zero time was $1.3 \mathrm{fmol} /$ culture for both conditions. The high ${ }^{125}$ I-Bgt 3.1 concentration necessitated the 1 min rinse to reduce nonspecific binding adequately for the "zero time" determinations. The curves represent least-squares regression fits of the data, assuming biphasic dissociation kinetics, as described in Figure 4. Similar results were obtained in a second experiment.

period $(10 \mathrm{~min})$ as well as the long period $(120 \mathrm{~min})$. The fact that nearly equivalent amounts of total specific binding were observed for the 2 labeling periods in sister cultures after a 1 min rinse indicates that the concentration was adequate. With the 10 min labeling, only $27 \%$ of the specific binding remained following a $2 \mathrm{hr}$ rinse. With the $120 \mathrm{~min}$ labeling, $41 \%$ of the toxin remained bound after a $2 \mathrm{hr}$ rinse (Fig. 5). The biphasic dissociation rate constants calculated for the different labeling times were nearly identical, but the proportion of slowly dissociating component increased from $33 \%$ after a 10 min incubation with ${ }^{125}$ I-Bgt 3.1 to $53 \%$ after a $120 \mathrm{~min}$ incubation (Table 1). Thus, Bgt 3.1 binding undergoes a time-dependent conversion to yield a form that has slow dissociation kinetics. Only a portion of the Bgt 3.1 binding appears to be converted to the slowly dissociating form under the conditions examined, however, since no increase in the proportion of slowly dissociating component was observed when the time of binding was increased from 60 to $120 \mathrm{~min}$ (Table 1). It is unlikely that this represents an equilibrium distribution of fast and slow components since most of the slow component forms within $10 \mathrm{~min}$, but the half-time for dissociation of the slow component is about 5 hr (Fig. 5, Table 1).

The possibility that the slowly dissociating form of bound ${ }^{125}$ I-Bgt 3.1 might represent toxin internalized by the cells was considered because previous studies demonstrated that one consequence of Bgt 3.1 interacting with $\alpha$-Bgt sites on the neurons

\begin{tabular}{|c|c|c|c|c|}
\hline $\begin{array}{l}{ }^{125} \text { I-Bgt } 3.1 \\
\text { concentration } \\
\text { (nM) }\end{array}$ & $\begin{array}{l}\text { Incubation time } \\
\text { (min) }\end{array}$ & $\begin{array}{l}k_{\mathrm{f}} \\
\left(\min ^{-1}\right)\end{array}$ & $\begin{array}{l}k_{\mathrm{s}} \\
\left(\min ^{-1}\right)\end{array}$ & $\begin{array}{l}\text { Slow component } \\
\text { (\% of total) }\end{array}$ \\
\hline 30 & 10 & 0.06 & 0.002 & 33 \\
\hline 10 & 60 & 0.05 & 0.002 & 54 \\
\hline 30 & 120 & 0.05 & 0.002 & 53 \\
\hline
\end{tabular}

Ciliary ganglion cultures were incubated in the indicated concentration of ${ }^{125} \mathrm{I}-\mathrm{Bgt} 3.1$ with $10^{-6} \alpha$-Bgt at $37^{\circ} \mathrm{C}$ for the indicated time, rinsed, and examined for dissociation of specifically bound ${ }^{225}$ I-Bgt 3.1. The dissociation rate constants for the fast $\left(k_{\mathrm{f}}\right)$ and slow $\left(k_{\mathrm{s}}\right)$ components and the proportion of slow component at zero time were estimated as described in Figures 4 and 5. Data are compiled from 2 separate experiments for each incubation time. 


\begin{tabular}{|c|c|c|c|}
\hline \multirow{2}{*}{$\begin{array}{l}\text { Experi- } \\
\text { ment }\end{array}$} & \multirow[b]{2}{*}{ Treatment } & \multicolumn{2}{|c|}{${ }^{125} \mathrm{I}-\mathrm{Bgt} 3.1$ bound } \\
\hline & & fmol/culture & $\%$ \\
\hline \multirow[t]{4}{*}{ A } & Control & $1.39 \pm 0.15$ & 100 \\
\hline & $2 \mathrm{hr}$ rinse & $0.62 \pm 0.01$ & 45 \\
\hline & DNP & $1.28 \pm 0.25$ & 92 \\
\hline & $\mathrm{DNP}+2 \mathrm{hr}$ rinse & $0.51 \pm 0.05$ & 37 \\
\hline \multirow[t]{5}{*}{ B } & Control & $1.58 \pm 0.25$ & 100 \\
\hline & $2 \mathrm{hr}$ rinse & $0.73 \pm 0.05$ & 46 \\
\hline & $2 \mathrm{hr}$ rinse $/$ acid & $0.31 \pm 0.04$ & 20 \\
\hline & $\mathrm{DNP}+2 \mathrm{hr}$ rinse & $0.79 \pm 0.03$ & 50 \\
\hline & $\mathrm{DNP}+2 \mathrm{hr}$ rinse/acid & $0.37 \pm 0.06$ & 23 \\
\hline
\end{tabular}

In experiment $A$, ciliary ganglion cultures were labeled for $1 \mathrm{hr}$ at $37^{\circ} \mathrm{C}$ with $10^{-8}$ $\mathrm{M}^{125} \mathrm{I}$-Bgt 3.1 in the presence of $10^{-6} \mathrm{M} \alpha$-Bgt and then rinsed for $10 \mathrm{sec}$ (control) or for $2 \mathrm{hr}\left(2 \mathrm{hr}\right.$ rinse) at $37^{\circ} \mathrm{C}$ and examined for specific binding of ${ }^{125} \mathrm{I}-\mathrm{Bgt} 3.1$ as described in Materials and Methods. DNP at $1 \mathrm{~mm}$ was present as indicated, starting $30 \mathrm{~min}$ prior to labeling and continuing through either the $10 \mathrm{sec}$ (DNP) or $2 \mathrm{hr}$ (DNP $+2 \mathrm{hr}$ rinse) rinse. In experiment $\mathrm{B}$, cultures were treated as described in A except that after rinsing, all cultures except controls were incubated $15 \mathrm{~min}$ on ice in rinse buffer ( $2 \mathrm{hr}$ rinse, and DNP $+2 \mathrm{hr}$ rinse) or in mild acid rinse (2 $\mathrm{hr}$ rinse/acid, DNP $+2 \mathrm{hr}$ rinse/acid) before determining specifically bound ${ }^{125} \mathrm{I}-$ Bgt 3.1. A repetition of experiment $A$ and 2 repetitions of experiment $B$ yielded similar results.

at $37^{\circ} \mathrm{C}$ is the internalization of $\alpha$-Bgt bound at subsaturating levels to the cells (Ravdin et al., 1981). No evidence was availablc on possible internalization at the Bgt 3.1-unique sitcs from this earlier study. Two approaches were taken here. In the first, 2,4-dinitrophenol (DNP) was used as an energy blocker to decrease ATP synthesis and thereby inhibit energy-requiring processes such as receptor-mediated endocytosis (Haigler et al., 1980). DNP was found to have no effect on the total amount of specific ${ }^{125} \mathrm{I}-\mathrm{Bgt} 3.1$ binding and no effect on the proportion retained by the cells after $2 \mathrm{hr}$ of rinsing (Table 2 ). The second approach took advantage of the finding that mild acid rinsing releases surface-bound protein ligands, leaving only internalized ligand associated witn the cells (Ascoli, 1982; Mahan et al., 1985). Mild acid treatment of labeled cultures after $2 \mathrm{hr}$ of normal rinsing removed $58 \%$ of the ${ }^{125} \mathrm{I}$-Bgt 3.1 associated with the cells. The fraction remained unchanged for cells labeled and rinsed in the presence of DNP (Table 2). These results argue against internalization being the major contributor to the amount of slowly dissociating component formed.

\section{Pharmacology of binding}

The pharmacology of the Bgt 3.1-unique sites displayed a pattern expected for nicotinic AChRs of ciliary ganglion neurons. Competition binding studies were carried out between ${ }^{125} \mathrm{I}-\mathrm{Bgt}$ 3.1 and various cholinergic agonists and antagonists over a range of concentrations. $\alpha$-Bgt at $10^{-6} \mathrm{M}$ was included to prevent ${ }^{125} \mathrm{I}-$ Bgt 3.1 binding to $\alpha$-Bgt sites. All of the specific ${ }^{125}$ I-Bgt 3.1 binding defined by inhibition with an excess of unlabeled Bgt 3.1 under these conditions was also blocked by carbachol and by $d$-tubocurarine (Fig. 6). The apparent inhibition constants $\left(K_{\mathrm{i}} \mathrm{s}\right)$ for a variety of cholinergic ligands were calculated from experiments as shown in Figure 6, and the values are listed in Table 3. Cholinergic agonists and antagonists both appeared to interact with a single class of Bgt 3.1 binding sites under equilibrium conditions since the Hill coefficients were all near unity (Table 3).

\section{Tissue distribution of binding}

${ }^{125}$ I-Bgt 3.1-unique sites were found on cell types expected to have neuronal nicotinic AChRs. High levels of binding were observed in cultures of ciliary ganglion neurons and sympathetic neurons from chick (Fig. 7). Very little if any specific binding

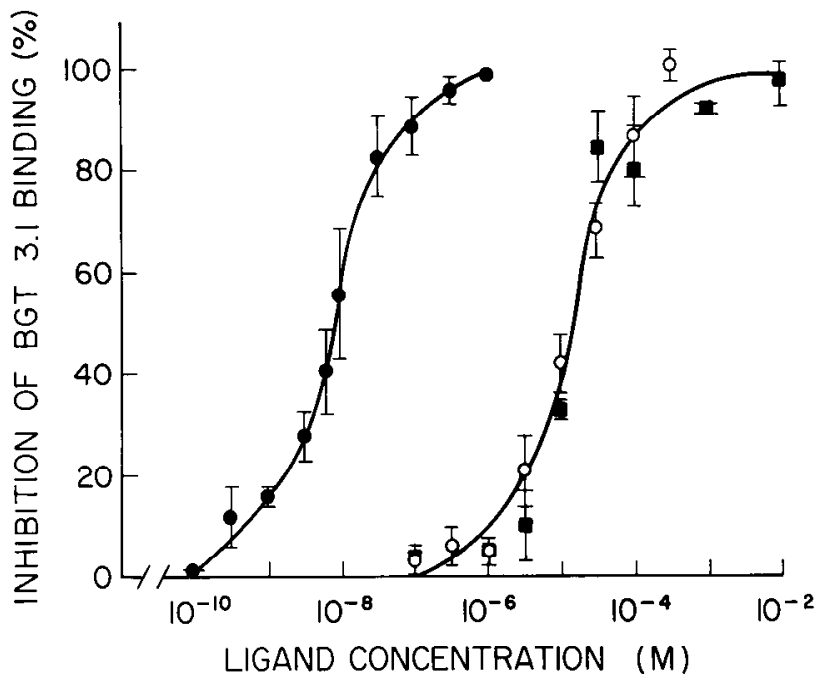

Figure 6. Inhibition of Bgt 3.1 binding by nicotinic cholinergic ligands. Binding of $10^{-8} \mathrm{M}^{125} \mathrm{I}-\mathrm{Bgt} 3.1$ in ciliary ganglion cultures was carried out at $37^{\circ} \mathrm{C}$ in the presence of $10^{-6} \mathrm{M} \alpha$-Bgt and the indicated concentrations of unlabeled Bgt 3.1 (filled circles), d-tubocurarine (open circles), or carbachol (filled squares). Results are expressed as a percentage of the maximum inhibition of ${ }^{125} \mathrm{I}-\mathrm{Bgt} 3.1$ binding caused by $10^{-6} \mathrm{M} \mathrm{Bgt}$ 3.1, defined as specific binding (see Materials and Methods). Values represent the mean \pm SE of determinations from 3 experiments each in the case of Bgt 3.1 and carbachol, and 5 experiments in the case of $d$-tubocurarine.

was found in cultures of skeletal myotubes or heart cells. Low levels were found in cultures of spinal cord cells and DRG neurons. In all cases, $10^{-6} \mathrm{M} \alpha$-Bgt was present throughout the incubation to block binding of ${ }^{125} \mathrm{I}$-Bgt 3.1 to possible $\alpha$-Bgt sites in the cultures, such as those associated with nicotinic AChRs on skeletal myotubes.

\section{Discussion}

The present findings demonstrate that ${ }^{125} \mathrm{I}-\mathrm{Bgt} 3.1$ recognizes 2 classes of binding sites on chick ciliary ganglion neurons in cell culture. The first class of sites appears to represent the highaffinity $\alpha$-Bgt sites previously described on the cells (Ravdin and Berg, 1979; Ravdin et al., 1981) since $\alpha$-Bgt blocks ${ }^{125}$ I-Bgt 3.1 binding in this case. The results corroborate earlier competition

\begin{tabular}{|c|c|c|}
\hline Agent & $\begin{array}{l}\text { Apparent } K_{\mathrm{I}} \\
(\mu \mathrm{M})\end{array}$ & $\begin{array}{l}\text { Hill coefficient } \\
\left(n_{\mathrm{H}}\right)\end{array}$ \\
\hline \multicolumn{3}{|l|}{ Agonists } \\
\hline Carbachol & $6.5 \pm 0.5$ & $0.9 \pm 0.2$ \\
\hline Nicotine & $3.1 \pm 0.8$ & $1.0 \pm 0.2$ \\
\hline $\mathrm{ACh}$ & $0.25 \pm 0.9$ & $0.9 \pm 0.1$ \\
\hline \multicolumn{3}{|l|}{ Antagonists } \\
\hline$d$-Tubocurarine & $8.3 \pm 3.2$ & $0.9 \pm 0.1$ \\
\hline Trimethaphan & $9.3 \pm 3.9$ & $0.9 \pm 0.1$ \\
\hline Bgt 3.1 & $0.003 \pm 0.001$ & $1.0 \pm 0.1$ \\
\hline
\end{tabular}

Apparent inhibitory binding constants $\left(K_{i} s\right)$ were calculated as described by Cheng and Prusoff (1973) for a series of cholinergic ligands using data from competition binding studies with ${ }^{125} \mathrm{I}$-Bgt 3.1 as described in Figure 6. In the case of $\mathrm{ACh}, 10^{-5}$ $M$ neostigmine was included in the binding reactions to prevent hydrolysis of the agonist. Hill coefficients $\left(n_{\mathrm{H}}\right)$ were determined by transforming the data to Hill plots. Values represent the mean \pm SE of 5 separate experiments for $d$-tubocurarine, and 3 for each of the others. 


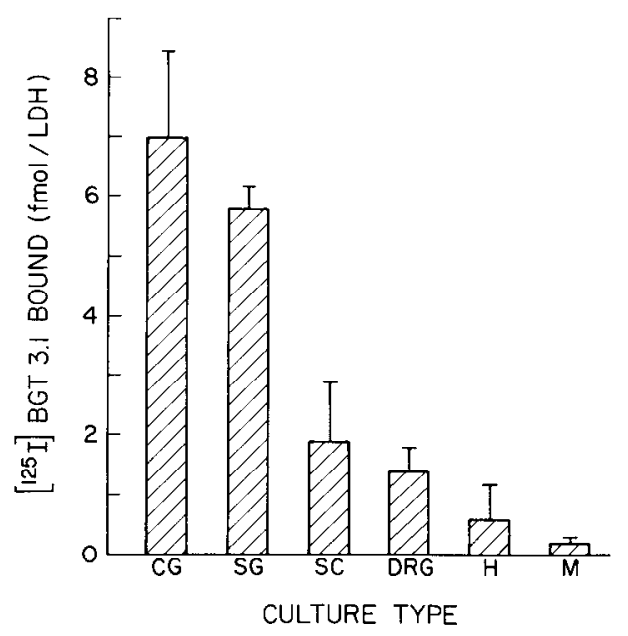

Figure 7. Cell types and Bgt 3.1 binding. The specific binding of $10^{-8}$ ${ }_{M}{ }^{125} I$-Bgt 3.1 in the presence of $10^{-6} \mathrm{M} \alpha$-Bgt was determined for a 1 $\mathrm{hr}$ incubation at $37^{\circ} \mathrm{C}$ for each of the indicated types of cell cultures. Results are expressed as fmol bound ${ }^{25}$ I-Bgt 3.1 per unit of LDH activity measured in sister cultures. Values represent the mean \pm SE of 8 determinations with triplicate cultures for ciliary ganglion $(C G)$ cultures, 5 for spinal cord $(S C)$ cultures, 4 each for dorsal root ganglion $(D R G)$, heart $(H)$, and sympathetic ganglion $(S G)$ cultures, and 3 for skeletal muscle $(M)$ cultures. Data were compiled from 8 separate culture platings for ciliary ganglion cultures, 3 for spinal cord cultures, and 2 in all other cases.

studies with unlabeled Bgt 3.1 and ${ }^{125} \mathrm{I}-\alpha-\mathrm{Bgt}$ that led to the same conclusion (Ravdin et al., 1981).

The second class of ${ }^{125} \mathrm{I}-\mathrm{Bgt} 3.1$ binding sites on the neurons has the properties expected for the functional nicotinic AChR on the cells. The $K_{\mathrm{d}}$ for ${ }^{125} \mathrm{I}$-Bgt 3.1 binding and the rates of association and dissociation of the labeled toxin from the cells are all consistent with the Bgt 3.1 conditions previously reported to block the $\mathrm{ACh}$ response of the neurons in culture (Ravdin and Berg, 1979). Moreover, ${ }^{125}$ I-Bgt 3.1 binding to this second class of sites is not inhibited by $\alpha$-Bgt and yet is inhibited both by cholinergic agonists and antagonists. The $K_{\mathrm{i}}$ for $d$-tubocurarine at the site is consistent with $d$-tubocurarine concentrations previously reported to block the ACh sensitivity of the neurons (Margiotta and Berg, 1982; Ravdin and Berg, 1979) and to block the $\mathrm{ACh}$-induced ${ }^{137} \mathrm{Ce}^{+}$flux of the cells in culture (Messing et al., 1984). The $K_{\mathrm{i}}$ values for $\mathrm{ACh}$ and carbachol indicate binding affinities that are $10^{2}-10^{3}$ times greater than the values estimated from physiological dose-response curves for agonist activation of the AChRs on the neurons in culture (Messing et al., 1984; Ogden et al., 1984; Smith et al., 1983). This may reflect an agonist-induced desensitization of the neuronal AChR analogous to that found with Torpedo electric organ AChR, where a 300-fold increase in agonist affinity can be observed as a result of desensitization (Weiland and Taylor, 1979). Studies on membrane fragments and detergent extracts from avian and mammalian brain have produced a range of values for the binding affinities of cholinergic ligands (Marks and Collins, 1982; Romano and Goldstein, 1980; Schneider et al., 1985; Whiting and Lindstrom, 1986b). The reported antagonist affinities are generally similar to those described here, but the agonist affinities vary up to a 100 times greater than those obtained here. The differences may be due to the preparations used (whole cells versus membrane fragments and solubilized receptor) or the receptor sources (brain versus autonomic AChRs).

The distribution of cell types having the second type of ${ }^{125} \mathrm{I}-$ Bgt 3.1 binding sites, i.e., toxin sites not recognized by $\alpha$-Bgt, is again consistent with the sites representing neuronal nicotinic
AChRs. Highest levels of binding were found with ciliary ganglion and sympathetic neurons, while little occurred with skeletal myotubes or heart cells. ${ }^{125} \mathrm{I}-\mathrm{Bgt} 3.1 \mathrm{did}$ bind to skeletal myotubes in culture, but the binding was completely blocked by $\alpha$-Bgt, indicating that it represented binding to the muscle AChR (S. Halvorsen and D. Berg, unpublished results). The low levels of binding observed with spinal cord and DRG neurons in culture are interesting and deserve additional study. DRG neurons derive developmentally from neural crest cells, as do ciliary ganglion neurons and sympathetic neurons. Precedence exists for sensory neurons expressing functional AChRs under certain conditions (Baccaglini and Cooper, 1982; Morita and Katayama, 1984).

The equilibrium binding studies with ${ }^{125} \mathrm{I}-\mathrm{Bgt} 3.1$ and the competition binding studies with cholinergic ligands all indicate that the high-affinity ${ }^{125} \mathrm{I}-\mathrm{Bgt} 3.1$ sites that are resistant to $\alpha$-Bgt on ciliary ganglion neurons behave as a homogeneous population of sites. Also, the association kinetics for ${ }^{125} \mathrm{I}-\mathrm{Bgt} 3.1$ binding are monophasic, as expected for a single class of sites. The dissociation kinetics, however, are multiphasic and reveal a time-dependent appearance of a slow component. The slow component is most likely a conversion product of the fast component. This conclusion follows from the observation that increasing the time of binding with $30 \mathrm{nM}{ }^{125} \mathrm{I}-\mathrm{Bgt} 3.1$ from 10 to 120 min produced no increase in the total amount of specific binding but did produce an increase in the proportion of bound ${ }^{125}$ I-Bgt 3.1 in the slowly dissociating form. Taken together, the equilibrium and kinetic data support a sequential model for Bgt 3.1 binding in which initially the toxin-receptor complex dissociates rapidly but is then converted over time to a state that dissociates slowly. Time-dependent multiphasic dissociation kinetics have also been observed for other ligand-receptor interactions, including the $\alpha$-Bgt-AChR complex from electric organ (Hess et al., 1975), the quinuclidinyl benzilate-muscarinic AChR complex in chick heart (Galper et al., 1977), and the thyrotropinreleasing hormone-receptor complex in rat brain (Johnson et al., 1984).

A simple reversible equilibrium for interconversion of fast and slow states, however, is inadequate to describe fully the Bgt 3.1 interactions with AChRs on ciliary ganglion neurons, since only about half of the toxin-receptor complex appears to undergo conversion to a slowly dissociating form under the conditions examined. These proportions would predict an equilibrium constant of approximately 1 for the conversion, but the apparent rates of appearance and disappearance of the slow form are incompatible with this. Accordingly, the results imply that either an inherent difference exists in the 2 types of binding initially (perhaps analogous to the 2 nonequivalent toxin-binding sites on muscle AChRs) or that a limitation is imposed on the conversion by some cellular function. Bgt 3.1 has previously been shown over the short term to induce the loss of about half of the binding sites on the neurons for anti-MIR mAbs (Smith et al., 1986). Since the MIR is thought to be associated with AChRs on the neurons (see below), this raises interesting questions about the relationship of the slow and fast components of Bgt 3.1 binding to the altered MIR sites. Internalization or sequestration of the bound ${ }^{125}$ I-Bgt 3.1 inside the cell seems unlikely to account for at least the bulk of the slowly dissociating component since formation of the component was not inhibited by treating the neurons with an energy blocker, and mild acid rinsing was able to remove rapidly the majority of bound radioactivity comprising the slow component. Binding studies with membrane fragments or solubilized receptor may help elucidate the mechanism by which the slow component is formed.

Previous studies have identified a class of $\alpha$-neurotoxin sites in intact chick ciliary ganglia that is distinct from $\alpha$-Bgt sites in the tissue (Chiappinelli, 1983; Loring et al., 1984). The $\alpha$-neurotoxins used in those cases, i.e., $\kappa$-bungarotoxin (Chiappinelli, 
1983) and toxin F (Loring ct al., 1984), have since been shown by electrophoretic and amino acid sequence analyses to be indistinguishable from Bgt 3.1 (Loring et al., 1986). $K_{\mathrm{d}}$ and $B_{\max }$ values were not given for the ganglionic sites, but the concentrations of toxin used and the amounts of binding observed were comparable to those reported here. Preliminary pharmacological characterization of the sites, however, indicated that while bromoacetylcholine did block $\kappa$-bungarotoxin binding, $d$-tubocurarine was "not very effective at blocking" the binding (Chiappinelli, 1983). Both $d$-tubocurarine and carbachol were reported to have no effect on toxin $F$ binding to what was presumably the same class of sites (Loring et al., 1984). The failure of common nicotinic ligands to block ganglionic binding is surprising in view of the present findings in cell culture and may reflect the difficulties of doing competitive binding studies on intact ganglionic tissue.

mAbs to the MIR of muscle and electric organ AChR $\alpha$-subunit have previously been used to identify a putative nicotinic AChR on chick ciliary ganglion neurons. The binding component has a predominantly synaptic location (Jacob et al., 1984) and has many of the biochemical and regulatory properties expected for the receptor (Smith et al., 1985, 1986). Bgt 3.1 can modulate the number of MIR-like sites on the neurons in culture (Smith et al., 1986). A preliminary report suggests that Bgt 3.1 sites in the ganglion (not blocked by $\alpha$-Bgt) have the same surface distribution (Loring and Zigmond, 1985) as previously reported for anti-MIR mAb sites (Jacob et al., 1984). Bgt 3.1 as a probe for the neuronal AChR has the power of directly associating $\mathrm{AChR}$ function with the binding component as well as permitting a pharmacological characterization of the binding site. A demonstration that Bgt 3.1 and anti-MIR mAbs bind to the same membrane component would corroborate previous evidence that the MIR-like antigen is part of the neuronal nicotinic AChR and would encourage use of both Bgt 3.1 and anti-MIR mAbs as neuronal AChR probes.

The number of AChRs identified on ciliary ganglion neurons in culture using ${ }^{125} \mathrm{I}-\mathrm{Bgt} 3.1$ is comparable to the number of antiMIR mAb binding sites reported previously for the cells (Smith et al., 1986), but the exact stoichiometry of binding and whether the probes bind to the same membrane component have yet to be determined. Interestingly, in the one case where the number of anti-MIR binding sites on the neurons clearly fails to change in parallel with developmental changes in the levels of $\mathrm{ACh}$ response associated with the cells (Margiotta et al., 1985, and unpublished results; Smith et al., 1986), the number of AChRs as determined by ${ }^{125} \mathrm{I}-\mathrm{Bgt} 3.1$ binding agrees with the number of $\mathrm{mAb}$ sites rather than the levels of ACh sensitivity (S. Halvorsen and D. Berg, unpublished observations). This apparent discrepancy between the number of total receptors inferred from the number of $\mathrm{Bgt} 3.1$ or $\mathrm{mAb}$ binding sites versus the relative levels of functional receptors inferred from the physiological studies is likely to have significance for mechanisms regulating AChR function on neurons.

Bgt 3.1 has a number of interesting properties. It binds to and reversibly blocks the function of AChRs on ciliary ganglion neurons (Ravdin and Berg, 1979). It can apparently modulate the number of AChRs on the cells, as reflected by changes induced in the number of anti-MIR mAb binding sites (Smith et al., 1986). Bgt 3.1 also recognizes the $\alpha$-Bgt binding sites on the neurons and can induce internalization of $\alpha$-Bgt bound to the cells when the $\alpha$-Bgt is present in subsaturating amounts on the sites (Ravdin et al., 1981). In addition, Bgt 3.1 can bind to the nicotinic AChRs of muscle and electric organ (Chiappinelli, 1983; Loring et al., 1984; S. Halvorsen and D. Berg, unpublished results). Some of these unusual properties, e.g., the modulation and internalization effects seen with ciliary ganglion neurons, may in part depend on the reported ability of Bgt 3.1 to dimerize in solution (Chiappinelli and Lee, 1985). The binding studies with ${ }^{125}$ I-Bgt 3.1 reported here for neurons confirm previous physiological studies on the specificity of Bgt 3.1: It appears to interact with only 2 major classes of high-affinity binding sites, these being the nicotinic AChR and the $\alpha$-Bgt binding component. As a result Bgt 3.1 is likely to be a useful probe for examining $A C h R$ regulation on neurons.

\section{References}

Ascoli, M. (1982) Internalization and degradation of receptor-bound human choriogonadotropin in Leydig tumor cells. J. Biol. Chem. 257: 13306-13311.

Baccaglini, P. I., and E. Cooper (1982) Influences on the expression of acetylcholine receptors on rat nodose neurones in cell culture. $J$. Physiol. (Lond.) 324: 441-451.

Berg, D. K. (1978) Acetylcholine synthesis by chick spinal cord neurons in dissociated cell culture. Dev. Biol. 66:500-512.

Breer, H., R. Kleene, and G. Hinz (1985) Molecular forms and subunit structure of the acetylcholine receptor in the central nervous system of insects. J. Neurosci. 5: 3386-3392.

Cheng, Y.-C., and W. H. Prusoff (1973) Relationship between the inhibition constant $\left(K_{\mathrm{I}}\right)$ and the concentration of inhibitor which causes $50 \%$ inhibition $\left(\mathrm{I}_{50}\right)$ of an cnzymatic reaction. Biochem. Pharmacol. 22: 3099-3108.

Chiappinelli, V. (1983) Kappa-bungarotoxin: A probe for the neuronal nicotinic receptor in the avian ciliary ganglion. Brain Res. 277: 921.

Chiappinelli, V., and J. C. Lee (1985) $\kappa$-Bungarotoxin: Self-association of a neuronal nicotinic receptor probe. J. Biol. Chem. 260: 61826186.

Conti-Tronconi, B. M., S. M. J. Dunn, E. A. Barnard, J. O. Dolly, F. A. Lai, N. Ray, and M. A. Raftery (1985) Brain and muscle nicotinic acetylcholine receptors are different but homologous proteins. Proc. Natl. Acad. Sci. USA 82: 5208-5212.

Fields, J. Z., W. R. Roeske, E. Morkin, and H. I. Yamamura (1978) Cardiac muscarinic cholinergic receptors: Biochemical identification and charactcrization. J. Biol. Chcm. 253: 3251-3258.

Galper, J. B., W. Klein, and W. A. Catterall (1977) Muscarinic acetylcholine receptors in developing chick heart. J. Biol. Chem. 252: 8692-8699.

Haigler, H. T., F. R. Maxfield, M. C. Willingham, and I. Pastan (1980) Dansylcadaverine inhibits internalization of 125 I-epidermal growth factor in BALB 3T3 cells. J. Biol. Chem. 255: 1239-1241.

Hess, G. P., J. E. Bulger, J. L. Fu, E. F. Hindy, and R. J. Silberstein (1975) Allosteric interactions of the membrane-bound acetylcholine receptor: Kinetic studies with $\alpha$-bungarotoxin. Biochem. Biophys. Res. Commun. 64: 1018-1027.

Jacob, M. H., and D. K. Berg (1983) The ultrastructural localizaion of $\alpha$-bungarotoxin binding sites in relation to synapses on chick ciliary ganglion neurons. J. Neurosci. 3: 260-271.

Jacob, M. H., D. K. Bcrg, and J. M. Lindstrom (1984) Shared antigenic determinant between the Elecrophorus acetylcholine receptor and a synaptic component on chick ciliary ganglion neurons. Proc. Natl. Acad. Sci. USA 81: 3223-3227.

Johnson, W. A., N. M. Nathanson, and A. Horita (1984) Solubilization and characterization of thyrotropin-releasing hormone receptors from rat brain. Proc. Natl. Acad. Sci. USA 81: 4227-4231.

Kemp, G., L. Bentley, M. G. McNamee, and B. J. Morley (1985) Purification and characterization of the $\alpha$-bungarotoxin binding protein from rat brain. Brain Res. 347: 274-283.

Lindstrom, J., B. Einarson, and S. Tzartos (1981) Production and assay of antibodies to acetylcholine receptors. Methods Enzymol. 74: 432-459.

Loring, R. H., and R. E. Zigmond (1985) Amino acid sequence of a ncurotoxin that blocks ncuronal nicotinic receptors and the localization of its binding sites in chick ciliary ganglion. Soc. Neurosci. Abstr. 11: 92.

Loring, R. H., V. A. Chiappinelli, R. E. Zigmond, and J. B. Cohen (1984) Characterization of a snake venom neurotoxin which blocks nicotinic transmission in the avian ciliary ganglion. Neuroscience 11: 989-999.

Loring, R. H., D. Andrews, W. Lane, and R. E. Zigmond (1986) Amino acid sequence of Toxin $F$, a snake venom that blocks neuronal nicotinic receptors. Brain Res. (in press).

Mahan, L. C., H. J. Motulsky, and P. A. Insel (1985) Do agonists 
promote rapid internalization of $\beta$-adrenergic receptors? Proc. Natl. Acad. Sci. USA 82: 6566-6570.

Margiotta, J. F., and D. K. Berg (1982) Functional synapses are established between ciliary ganglion neurons in dissociated cell culture. Nature 296: 152-154.

Margiotta, J. F., D. K. Berg, and V. E. Dionne (1985) Regulation of acetylcholine receptors on chick ciliary ganglion neurons in cell culture. Soc. Neurosci. Abstr. 11: 170.

Marks, M. J., and A. C. Collins (1982) Characterization of nicotine binding in mouse brain and comparison with the binding of $\alpha$-bungarotoxin and quinuclidinyl benzilate. Mol. Pharmacol. 22: 554-564.

Messing, A., B. Bizzini, and N. K. Gonatas (1984) Concanavalin A inhibits nicotinic acetylcholine receptor function in cultured chick ciliary ganglion neurons. Brain Res. 303: 241-249.

Mitsuka, M., and H. Hatanaka (1983) Selective loss of acetylcholine sensitivity in a nerve cell line cultured in hormone-supplemented serum-free medium. J. Neurosci. 3: 1785-1790.

Morita, K., and Y. Katayama (1984) Two types of acetylcholine receptors on the soma of primary afferent neurons. Brain Res. 290: 348-352.

Nathanson, N. M. (1983) Binding of agonists and antagonists to muscarinic acetylcholine receptors on intact cultured heart cells. J. Neurochem. 41: 1545-1549.

Nishi, R., and D. K. Berg (1977) Dissociated ciliary ganglion neurons in vitro: Survival and synapse formation. Proc. Natl. Acad. Sci. USA 74: 5171-5175.

Nishi, R., and D. K. Berg (1981) Two components from eye tissue that differentially stimulate the growth and development of ciliary ganglion ncurons in cell culture. J. Neurosci. 1: 505-513.

Ogden, D. C., P. T. A. Gray, D. Colquhoun, and H. P. Rang (1984) Kinetics of acetylcholine activated ion channels in chick ciliary ganglion neurones grown in tissue culture. Pfluegers Arch. 400: 44-50.

Patrick, J., and W. B. Stallcup (1977) Immunological distinction between acetylcholine receptor and the $\alpha$-bungarotoxin binding component on sympathetic neurons. Proc. Natl. Acad. Sci. USA 74: 46894692.
Ravdin, P. M., and D. K. Berg (1979) Inhibition of neuronal acetylcholine sensitivity by alpha-toxins from Bungarus multicinctus venom. Proc. Natl. Acad. Sci. USA 76: 2072-2076.

Ravdin, P. M., R. M. Nitkin, and D. K. Berg (1981) Internalization of $\alpha$-bungarotoxin on neurons induced by a neurotoxin that blocks neuronal acetylcholine sensitivity. J. Neurosci. 1: 849-861.

Romano, C., and A. Goldstein (1980) Stereospecific nicotine receptors on rat brain membranes. Science 210: 647-650.

Schneider, M., C. Adee, H. Betz, and J. Schmidt (1985) Biochemical characterization of two nicotinic receptors from the optic lobe of chick. J. Biol. Chem. 260: 14505-14512.

Smith, M. A., J. F. Margiotta, and D. K. Berg (1983) Differential regulation of acetylcholine sensitivity and $\alpha$-bungarotoxin binding sites on ciliary ganglion neurons in cell cultures. J. Neurosci. 3: 23952402.

Smith, M. A., J. Stollberg, J. M. Lindstrom, and D. K. Berg (1985) Characterization of a component in chick ciliary ganglia that crossreacts with monoclonal antibodies to muscle and electric organ acetylcholine receptor. J. Neurosci. 5: 2726-2731.

Smith, M. A., J. F. Margiotta, A. Franco, Jr., J. M. Lindstrom, and D. K. Berg (1986) Cholinergic modulation of an acetylcholine receptorlike antigen on the surface of chick ciliary ganglion neurons in cell culture. J. Neurosci. 6: 946-953.

Stollberg, J., P. J. Whiting, J. M. Lindstrom, and D. K. Berg (1986) Functional blockade of neuronal acetylcholine receptors by antisera to a putative receptor from brain. Brain Res. 378: 179-182.

Weiland, G., and P. Taylor (1979) Ligand specificity of state transitions in the cholinergic receptor: Behavior of agonists and antagonists. Mol. Pharmacol. 15: 197-212.

Whiting, P. J., and J. Lindstrom (1986a) Purification and characterization of an acetylcholine receptor from chick brain. Biochemistry 25: 2082-2093.

Whiting, P., and J. Lindstrom (1986b) Pharmacological properties of immuno-isolated neuronal nicotinic receptors. J. Neurosci. 6: 30613069 . 